\title{
Universal parameterization of initial-state fluctuations and its applications to event-by-event anisotropy
}

\author{
Li Yan ${ }^{\mathrm{a}}$, Jean-Yves Ollitrault ${ }^{\mathrm{a}}$, Art Poskanzer ${ }^{\mathrm{b}}$ \\ ${ }^{a}$ CNRS, URA2306, IPhT, Institut de Physique Théorique de Saclay, F-91191 Gif-sur-Yvette, France \\ ${ }^{b}$ Lawrence Berkeley National Laboratory, Berkeley, California, 94720
}

\begin{abstract}
We propose Elliptic Power and Power parameterizations for the probability distribution of initial state anisotropies in heavy-ion collisions. By assuming a linear eccentricity scaling, the new parameterizations can also be applied to fluctuations of harmonic flow. In particular, the recently measured flow multiparticle cumulants and event-by-event distributions are analyzed.
\end{abstract}

Keywords:

Heavy-ion collisions, fluctuations, anisotropic flow

\section{Introduction}

It was recently realized that the understanding of fluctuations, in particular fluctuations in the initial state, is an essential ingredient in the analyses of ultra-relativistic heavy-ion collisions [1]. To characterize a fluctuating initial state theoretically, effective models have been proposed by properly introducing fluctuations on top of nucleusnucleus collisions [2,3]. However, despite somewhat success of these models, initial state of heavy-ion collisions still contributes a major fraction of uncertainty to quantitative predictions [4, 5]. In experiments, initial state fluctuations can be revealed by the study of anisotropic flow $v_{n}$. Defined as the Fourier harmonics of the corresponding particle spectrum, $v_{n}$ reflects the property of bulk medium expansion, and its response to the initial state anisotropy. Taking into account thus the direct mapping between $v_{n}$ and initial anisotropy, which is commonly formulated as eccentricity $\varepsilon_{n}$, it is expected that event-by-event (EbyE) distribution of $v_{n}$ is largely determined by fluctuations of $\varepsilon_{n}$. Indeed, many non-trivial observations have been made in heavy-ion experiments regarding flow fluctuations, among which the EbyE distribution of $v_{n}$ in $\mathrm{Pb}-\mathrm{Pb}$ collisions [6], and cumulants of elliptic flow $v_{2}$ from $\mathrm{p}$ - $\mathrm{Pb}$ collisions [7, 8] will be discussed in this work. In this paper, without detailed modeling of initial state we propose a new parameterization to describe $\varepsilon_{n}$ fluctuations. As will be shown in Section 2, the crucial improvement of our new parameterization is rooted in the fact that $\left|\varepsilon_{n}\right| \leq 1$. The universality of parameterizing fluctuations of $\varepsilon_{n}$ will be addressed also in Section 2 . In Section 3 we apply the parameterization to the measured flow cumulants and flow distribution.

\section{Elliptic-Power and Power parameterizations}

Initial state eccentricity characterizes the spatial anisotropy of a system created in heavy-ion collisions. Ellipticity, for example, which can be defined in a complex form as $\varepsilon_{2} e^{i 2 \Phi_{2}}=\varepsilon_{2 x}+i \varepsilon_{2 y}=-\left\{r^{2} e^{i 2 \phi_{r}}\right\} /\left\{r^{2}\right\}$, characterizes the elliptic deformation. In the definition, $\{\ldots\}=\int d x d y \ldots \epsilon(x, y)$ stands for an average in the transverse plane with respect to energy density $\epsilon(x, y)$, which implies that the modeling of eccentricity replies on an effective description of 

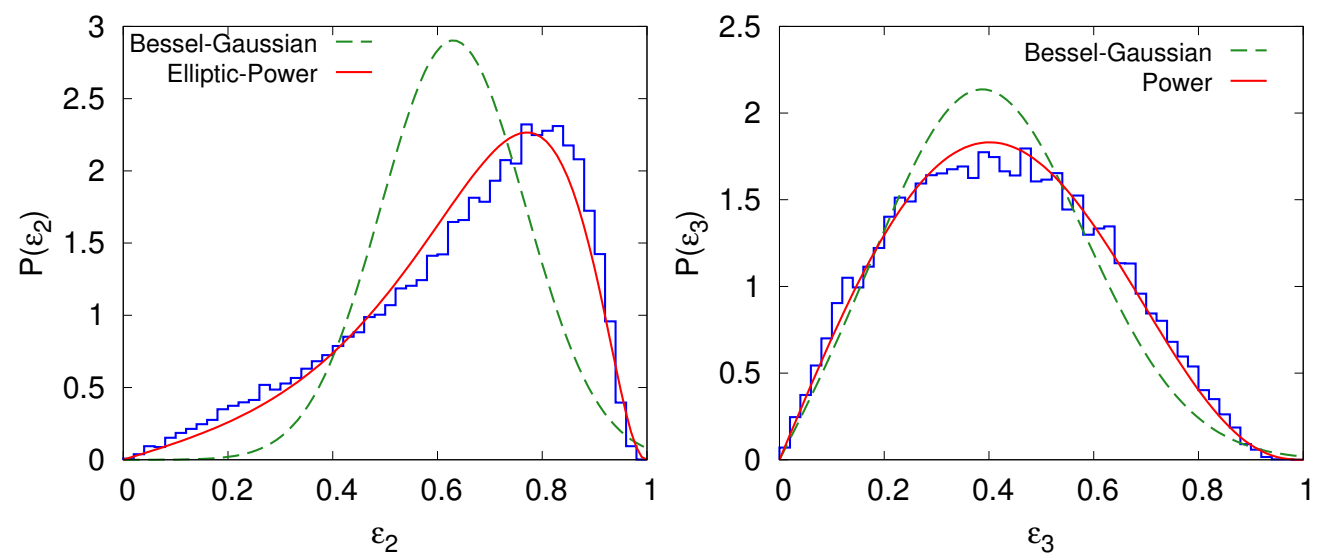

Figure 1. (Color online) Distribution of $\varepsilon_{2}$ and $\varepsilon_{3}$ in 75-80\% central Pb-Pb collisions. Histograms are obtained by PHOBOS Monte-Carlo Glauber simulations. Fit by Bessel-Gaussian parameterization (green dashed lines) are shown comparing to Elliptic Power (red solid line in the left panel) for $\varepsilon_{2}$ and Power (red solid line in the right panel) for $\varepsilon_{3}$.

energy deposition from nucleon-nucleon collisions. In addition, fluctuations and correlations in the colliding system need to be included as well, so that initial state eccentricity fluctuates on an EbyE basis. Nonetheless, taking into account fluctuations of nucleon as a dominant effect one arrives at the "independent source model". Nucleon-nucleon correlations are ignored in such a simplified description, but effects of fluctuation can be solved analytically. Following similar procedures taken in the original applications [9] and futher assuming, such as Gaussian background etc., for a system configured by $N$ independent point-like sources, the spatial anisotropy is found to fluctuate according to the Elliptic-Power distribution [10],

$$
P\left(\varepsilon_{x}, \varepsilon_{y}\right)=\frac{\alpha}{\pi}\left(1-\varepsilon_{0}^{2}\right)^{\alpha+\frac{1}{2}} \frac{\left(1-\varepsilon_{x}^{2}-\varepsilon_{y}^{2}\right)^{\alpha-1}}{\left(1-\varepsilon_{0} \varepsilon_{x}\right)^{2 \alpha-1}} .
$$

Eq. (1) contains two parameters. $\alpha \sim N$ is approximately determined by the magnitude of fluctuations. $\varepsilon_{0} \sim \varepsilon_{R P}$ is constrained by the event-averaged eccentricity, where $\varepsilon_{R P}$ is the generally defined reaction-plane ellipticity. For the cases when event-averaged energy density is azimuthally symmetric, such as proton-lead collisions carried out recently at $\mathrm{LHC}[7,8], \varepsilon_{0}=0$. Eq. (1) reduces to the Power distribution [11]

$$
P\left(\varepsilon_{x}, \varepsilon_{y}\right)=\frac{\alpha}{\pi}\left(1-\varepsilon_{x}^{2}-\varepsilon_{y}^{2}\right)^{\alpha-1} .
$$

Integrating out the angular dependence in Eqs. (1) and (2), we obtain 1-dimensional Elliptic-Power and Power parameterizations for EbyE fluctuating eccentricities. Note that integration of angle in Eq. (1) results in a hypergeometric function which can be done in practice numerically. We have tested both of these new parameterizations by comparing to the eccentricity distributions obtained by Monte-Carlo simulations of Glauber [2] and IP-Glasma [3] models, and reasonably good fits are found universally in all collision centralities [10]. In Fig. 1 we present one of such comparisons for collisions with centrality percentile $75-80 \%$. We notice that our new parameterizations respect the fact that $\varepsilon_{n}$ 's are bounded by unity. This crucial property leads to significant improvements comparing to Bessel-Gaussian distribution, especially for small systems where $\varepsilon_{n}$ are larger.

\section{Applications to $\mathrm{p}-\mathrm{Pb}$ and $\mathrm{Pb}-\mathrm{Pb}$ systems}

Medium response to the initial state of heavy-ion collisions converts spatial anisotropy into anisotropic flow. In particular, for elliptic flow $v_{2}$ and triangular flow $v_{3}$, it is known that the response is to a good approximation linear (see for instance [12]), namely, $v_{2} e^{i 2 \Psi_{2}}=\kappa_{2} \varepsilon_{2} e^{i 2 \Phi_{2}}$ and $v_{3} e^{i 3 \Psi_{3}}=\kappa_{3} \varepsilon_{3} e^{i 3 \Phi_{3}}$. The linear eccentricity scaling allows us to apply Eqs. (1) and (2) to flow fluctuations, after a recaling with flow response coefficient $\kappa_{2}$ or $\kappa_{3}$. 

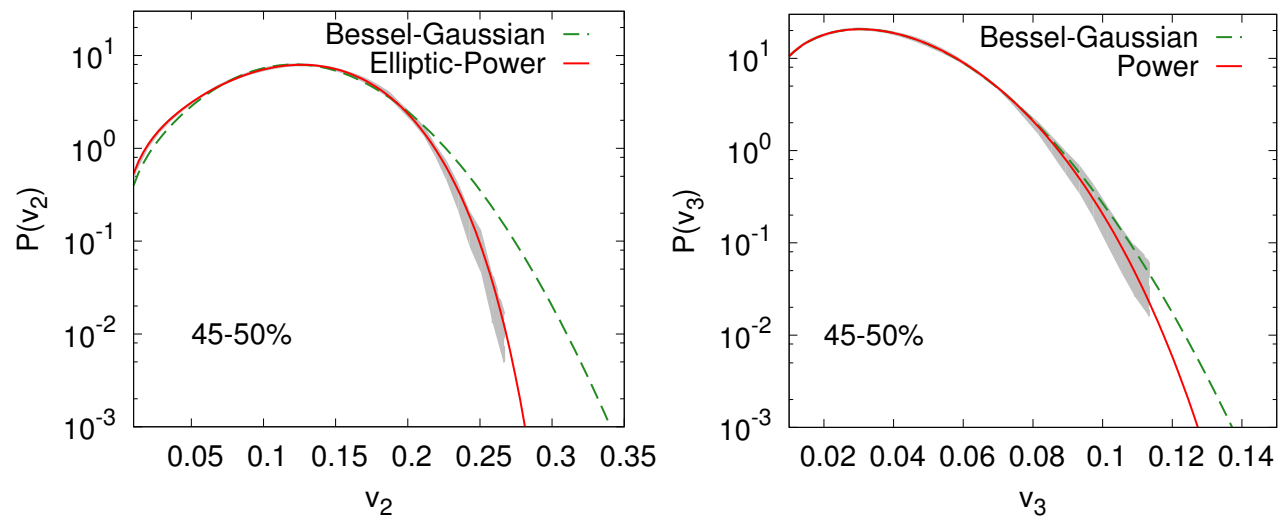

Figure 2. (Color online) Fits of ATLAS [6] flow event distribution (grey band) from $45-50 \%$ central Pb-Pb collisions, with Elliptic Power and Power (red solid lines) distributions. Bessel-Gaussian fits (green dashed lines) are shown as comparisons.

One application is the study of multiparticle cumulants of the measured flow. It should be emphasized that flow cumulants have non-trivial dependence on the nature of fluctuations. For example, one may check that for a pure Gaussian fluctuation, higher order flow cumulants vanish by definition. For the Power distribution in Eq. (2), we notice that cumulants of any order can be analytically expressed as a function of $\alpha$ [11]. In addition, it has also been found that higher order cumulants of Power distribution follow a certain pattern, which results in determined relations among cumulant ratios. Recent measurements by CMS collaboration [7, 8] confirms that $\left(v_{2}\{4\} / v_{2}\{2\}\right)$ and $\left(v_{2}\{6\} / v_{2}\{4\}\right)$ from proton-lead collisions are quantitatively consistent with Power distribution predictions, which strongly supports the picture of collective expansion in the $\mathrm{p}-\mathrm{Pb}$ system [11].

Alternatively flow fluctuations can be analyzed by fitting EbyE flow distribution with Elliptic-Power or Power parameterizations. Replacing $\varepsilon_{2}$ everywhere in Eq. (1) by $v_{2} / \kappa_{2}$, one finds a rescaled Elliptic-Power distribution, as a function of $\alpha, \varepsilon_{0}$ and $\kappa_{2}$. In a similar manner, for $v_{3}$ the substitution $\varepsilon_{3}=v_{3} / \kappa_{3}$ leads to rescaled Power distribution as a function of $\alpha$ and $\kappa_{3}$. In Fig. 2, we present fits of ATLAS measured EbyE $v_{2}$ and $v_{3}$ distribution from $45-50 \%$ centrality $\mathrm{Pb}-\mathrm{Pb}$ collisions [6], with Elliptic-Power and Power parameterizations. Comparing to Bessel-Gaussian, it is clear that both Elliptic-Power and Power achieve better agreement with experiments. Similar fits can be extended to all centrality bins, and as expected, we found that the improvements with Elliptic Power and Power parameterizations are more pronounced as centrality percentile grows. In Fig. 3 the extracted parameters from the fitting procedure are shown as a function of centrality. Shaded area of $\alpha_{2}, \epsilon_{0}$ and $\kappa_{2}$ are associated with systematic and statistical errors of the measured $v_{2}$, while for $\alpha_{3}$ and $\kappa_{3}$ only the effect of statistical errors is considered. We found that systematic errors of $v_{3}$ lead to anomalously large uncertainties of $\alpha_{3}$ and $\kappa_{3}$, which makes the results from ATLAS $v_{3}$ less meaningful. Nevertheless, we leave $\alpha_{3}$ and $\kappa_{3}$ in Fig. 3. It should be noticed that the way of obtaining these parameters, especially flow response coefficients $\kappa_{2}$ and $\kappa_{3}$, relies very little on the detailed modeling of initial states. The left panel of Fig. 3 shows a decrease of $\alpha$ with respect to centrality percentile, which indicates an increasing of initial state fluctuations from central to peripheral collisions. Also, we find that the average geometry is more elliptic towards peripheral collisions, as being depicted by the growth of $\epsilon_{0}$ in the middle of Fig. 3. Both of these results are consistent with our naïve understanding. We also plot in Fig. 3 the corresponding predictions by PHOBOS MC-Glauber and IP-Glasma models. Flow response coefficient $\kappa_{2}$ is solely determined by the bulk property of the medium. As shown in the right panel of Fig. 3, $\kappa_{2}$ has a clear trend of decreasing when the system is getting smaller, as anticipated by hydrodynamics. With a 2+1D viscous hydrodynamics (details of our hydrodynamics modeling can be found, for instance, in [13]), we found that $\kappa_{2}$ is well described as a function of shear viscosity over entropy ratio $\eta / s$,

$$
\kappa_{2}(\eta / s)=C_{0}\left[\kappa_{2}^{\text {ideal }}-\frac{\eta}{s} \delta \kappa_{2}\right]
$$

where $\delta \kappa_{2}=-\left[\kappa_{2}^{\text {ideal }}-\kappa_{2}^{\text {vise. }}\right] /(1 / 4 \pi)$ characterizes the change of response coefficients due to shear viscosity. $\kappa_{2}^{\text {ideal }}$ and $\kappa_{2}^{\text {visc. }}$ are obtained via hydrodynamics, with $\eta / s$ specified to be zero and $1 / 4 \pi$ respectively in simulations. Constant 

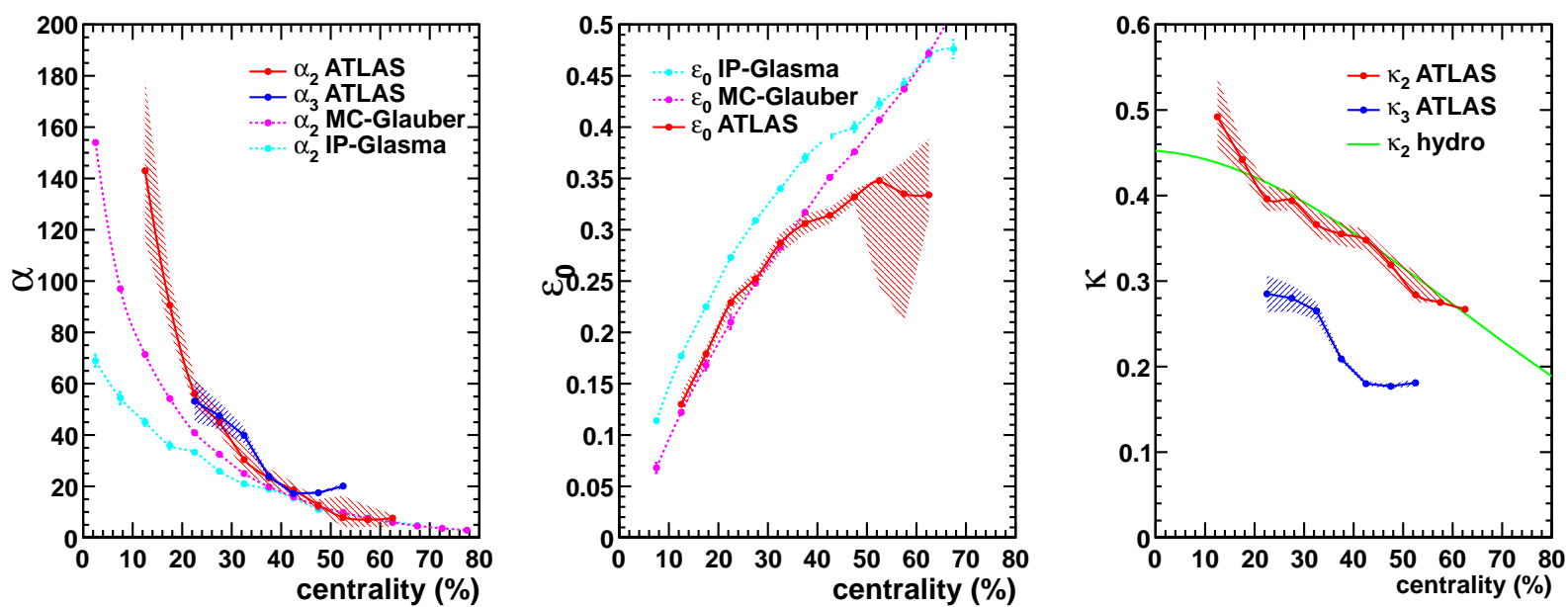

Figure 3. (Color online) Parameters extracted from fit of ATLAS $v_{2}$ event distribution. $\alpha$ and $\varepsilon_{0}$ from Glauber and IP-Glasma models are present for comparisons. $\kappa_{2}$ from hydro predictions with $\eta / s \sim 0.18$ is shown as green solid line in the right panel.

$C_{0} \sim 1.68$ in Eq. (3) takes into account all the extra effect that is not included in our hydrodynamic calculations. Eq. (3) returns an estimate of $\eta / s \sim 0.18$.

\section{Summary and conclusions}

We have proposed Elliptic Power and Power parameterizations for the initial eccentricity fluctuations. The validity of parameterizations are examined by fitting to effective models, with satisfactory agreements universally found. We further apply rescaled Elliptic Power and Power parameterizations to the distribution of anisotropic flow $v_{n}$, using the linear eccentricity scaling. Fitting to experiment data allows us to extract parameters which are relevant to the information of initial state, such as $\alpha$ and $\epsilon_{0}$. Also, and more importantly, flow response coefficient $\kappa_{2}$ is obtained quantitatively without any effective modeling of initial states, which provides a more self-contained way to estimate of the bulk property of quark-gluon plasma.

\section{Acknowledgments}

LY and JYO are funded by the European Research Council under the Advanced Investigator Grant ERC-AD267258. AMP was supported by the Director, Office of Science, of the U.S. Department of Energy.

\section{References}

[1] Alver B and Roland G 2010 Phys.Rev. C81 054905 (Preprint 1003.0194)

[2] Alver B, Baker M, Loizides C and Steinberg P 2008 (Preprint 0805.4411)

[3] Schenke B, Tribedy P and Venugopalan R 2014 (Preprint 1403.2232)

[4] Retinskaya E, Luzum M and Ollitrault J Y 2014 Phys.Rev. C89 014902 (Preprint 1311.5339)

[5] Luzum M and Ollitrault J Y 2013 Nucl.Phys. A904-905 377c-380c (Preprint 1210.6010)

[6] Aad G et al. (ATLAS Collaboration) 2013 JHEP 1311183 (Preprint 1305. 2942)

[7] Chatrchyan S et al. (CMS Collaboration) 2013 Phys.Lett. B724 213-240 (Preprint 1305.0609)

[8] Wang Q (CMS Collaboration) 2014 Talk at Quark Matter 2014

[9] Ollitrault J Y 1992 Phys.Rev. D46 229-245

[10] Yan L, Ollitrault J Y and Poskanzer A M 2014 (Preprint 1405.6595)

[11] Yan L and Ollitrault J Y 2014 Phys.Rev.Lett. 112082301 (Preprint 1312.6555)

[12] Niemi H, Denicol G, Holopainen H and Huovinen P 2013 Phys.Rev. C87 054901 (Preprint 1212.1008)

[13] Teaney D and Yan L 2014 Phys.Rev. C89 014901 (Preprint 1304.3753) 